\title{
Nanomechanical properties of imprinted amorphous NiAl alloys using atomic simulations
}

\author{
Te-Hua Fang ${ }^{a^{*}}$, Chao-Chun Huang ${ }^{b}$, Ming-Hong Lin ${ }^{c}, J^{\prime h-K a i ~ S u}{ }^{d}$, Ivo Stachive \\ Department of Mechanical Engineering, National Kaohsiung University of Applied Sciences, \\ Kaohsiung 80778, Taiwan \\ afang.tehua@msa.hinet.net, ${ }^{b}$ cchuang1942@gmail.com, \\ cmhlin@kuas.edu.tw, ${ }^{\mathrm{d}} 1103303142 @ g m . k u a s . e d u . t w,{ }^{\mathrm{e}}$ stachiv@kuas.edu.tw
}

\begin{abstract}
Keywords: Nanoimprinting process, Molecular dynamics; Amorphous NiAl; Deformation of NiAl.
\end{abstract}
\begin{abstract}
In this work, the molecular dynamics simulations are used to investigate the mechanical behavior of the imprinted nickel aluminide alloys. These atomic simulations account for the mold size, working temperature, imprinting velocity and the elastic recovery. It has been found that there exists a significant difference of the shear stain distribution in the inhomogeneous plastic deformation around the mold corner, whereas obtained lower elastic recovery values in the imprinting process indicates a higher material formability. Moreover, it has been also shown that the degree of the material softening increases with temperature causing the graduate extension of the strain to the bottom of the material. And, finally, the elastic recovery of the imprinted material decreases as the imprinting velocity is increased.
\end{abstract}

\section{Introduction}

Amorphous alloys have very good mechanical strength, toughness and fracture strength, therefore they are considered to be one of the most prospective materials for new generation of the tools, jet engines and recently also the microsystems [1]. Particularly, the nickel aluminide (NiAl) alloys have an excellent corrosion resistance at a high temperature and, as a result, they are often used as the main blades coatings [2]. It is worth noting that the phase equilibria for the $\mathrm{B} 2 \mathrm{NiAl}$ composition are different as the phase equilibria known for the amorphous two binary systems.

Micromold embossing and imprinting technologies are known to be easy accessible and low-cost techniques to fabricate the various micro components [3]. However, despite the fact that the plenty of experimental and numerical studies has been performed $[4,5]$, the quantitative evaluation of the nanoimprinting process of amorphous NiAl alloy is still not yet fully understood. In response, the purpose of this paper is to quantitatively study the mechanical properties of the amorphous NiAl alloy during the imprinting process utilizing the molecular dynamics (MD) simulations. Based on the results, impacts of mold size, imprinting rate and temperature on the elastic recovery are revealed.

\section{Modeling}

The nanoimprinting process of the amorphous NiAl alloy considered in the MD simulations consists of the mold diamond indenter, the NiAl amorphous specimen, and the thermostatic and fixed layers as shown in Fig. 1. In addition, to further simplify computations of the mechanics and patterns, the mold is assumed to be composed of the rigid-body atoms. Dimensions of the mold indenter are $15 \mathrm{~nm}$ in length, $3 \mathrm{~nm}$ in width and $4 \mathrm{~nm}$ in height, and dimensions of the specimen are $15 \mathrm{~nm}$ in length, $3 \mathrm{~nm}$ in width and $15 \mathrm{~nm}$ in height. Additionally, specimen comprises of 49770 $\mathrm{NiAl}$ atoms. A Cartesian coordinate system with the original point of the coordinate located in the center of the lower left atom of the specimen is used for computations. Motion of atoms in the thermostatic and Newtonian layers follows the Newton's second law. Furthermore, the velocity rescaling method is performed on the equilibration for 30 ps at $300 \mathrm{~K}$. Besides, the van der Waals interactions of $\mathrm{C}-\mathrm{Ni}$ and $\mathrm{C}-\mathrm{Al}$ are governed by Lennard-Jones potential [5] and the potential energy 
between $\mathrm{Ni}$ and $\mathrm{Al}$ atoms can be calculated utilizing the second-moment approximation of the many-body tight-binding (SMA-TB) equation [6]. To increase the computation efficiency the periodic boundary conditions are imposed on the $x$ and $y$ axes and, importantly, to have a structural stability of $\mathrm{Ni}-\mathrm{Al}$ atoms throughout the imprinting process, the lowest bottom layer of the substrate atoms has been fixed.

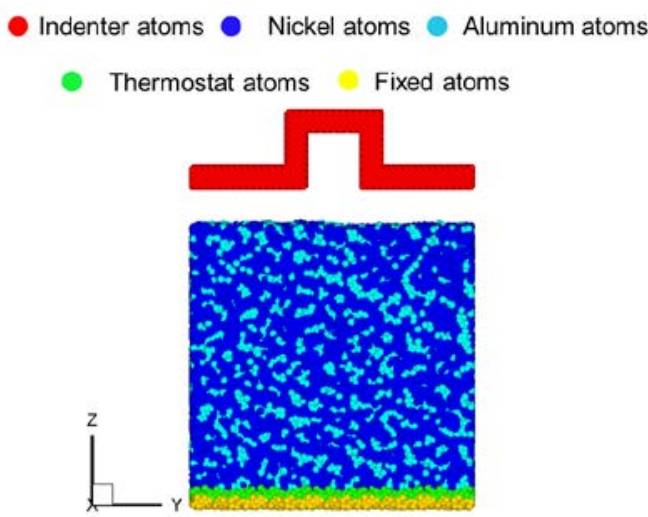

Figure 1. Sketch of the nanoimprinting process of the amorphous NiAl alloy considered in the MD simulations

\section{Results and discussion}

Figure 2 presents the amorphous $\mathrm{NiAl}$ alloy imprinted at the constant temperature of $300 \mathrm{~K}$ and a loading velocity of $30 \mathrm{~m} / \mathrm{s}$ for different mold sizes represented through the aspect ratio parameter $\lambda$. This parameter is defined as ratio of the mold height against its width. To describe and, consequently, to observe in details the atomic deformation in each strain field region, individual atoms of the specimen has been colored differently based on the shear strain distribution during the imprinting process. Namely, red color represents the largest shear strain, while the blue one stands for the lowest shear strain. For small values of $\lambda$ the cavity space increased and, correspondingly, the position of mold cavity sidewalls strain became smaller, i.e. there is sufficiently large space for the material to enter into the mold cavity (see Figs. 2(a) - 2(c)). When the aspect ratio is larger than 0.6 , then the cavity space is reduced resulting in the excessive compression around the sidewall of the mold. Nevertheless, for very large cavity sizes the material does not completely fill the cavity as shown in Fig 2(d).

In this work, the shear stain distribution is calculated as the difference in atom position before and after deformation. A significant difference in shear strain distribution for the inhomogeneous plastic deformation around the mold corner has been observed. Deformation of the amorphous NiAl alloys is obtained based on the shear transformation zone (STZ) formation [7]. These shear transformations are interrelated with the dislocations and the point defects. SZT zone and also the stress concentration are then revealed by existence of the local plasticity. 


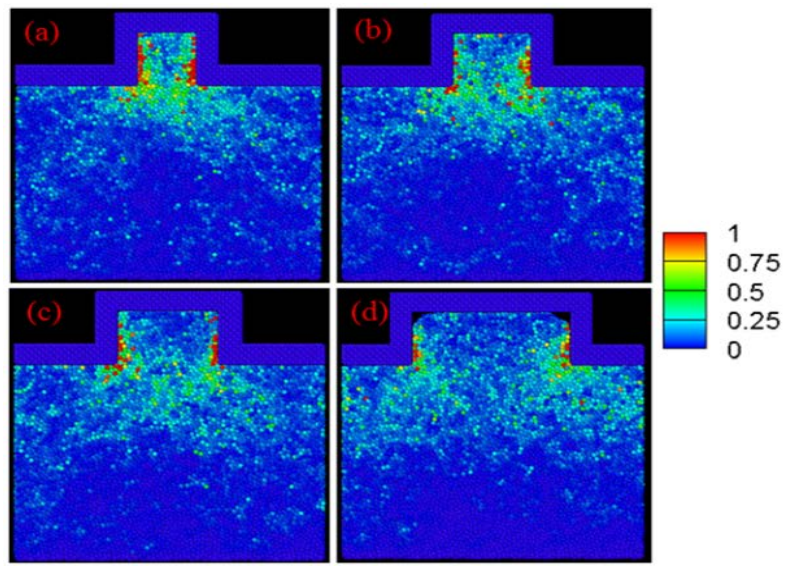

Figure 2. NiAl alloys imprinted at a temperature of $300 \mathrm{~K}$ and a loading velocity of $30 \mathrm{~m} / \mathrm{s}$ for various mold sizes of (a) $\lambda=1$, (b) $\lambda=0.75$, (c) $\lambda=0.6$ and (d) $\lambda=0.375$.

Variation of the loading force versus time step for various mold sizes during the imprinting process is presented in Fig. 3. The loading force curves can be separated in to the three stages of the imprinting process, i.e. loading, holding and unloading stages. Loading stage is characterized by a small negative value of the force at time period of about 30 ps caused by the der Waals attractive force action between the $\mathrm{Ni}-\mathrm{Al}$ and the mold. Then, at the time period of about $50-100 \mathrm{ps}$ the exerted load increases with time. Noticing only that for small aspect ratio $\lambda$, the material has a relatively larger space to be filled. It indicates that the interaction of mold atoms and contact area are also increased with time enabling to overcome the imprinted deformation of materials. The holding process is realized at a time period of $120-170$ ps and is characterized by small force fluctuations. These force fluctuations are related to the adjusting position of atoms to relax the stored strain energy of material. At the beginning of the unloading stage, the loading force suddenly falls to a low value in the range of $-300 \mathrm{nN}$, which is more likely attributed to the significantly larger adhesion between the mold and the materials. Figure 4 shows the elastic recoveries of NiAl alloys with different mold sizes at a temperature of $300 \mathrm{~K}$. The elastic recovery $\mathrm{\eta}$ is defined as the variation of the filled mold and the unloaded specimen [8]. It has been found that the height $\left(\mathrm{y}_{h}\right)$ and width $\left(y_{w}\right)$ recoveries increase when the aspect ratio of mold decrease. Here, a lower elastic recovery values in the imprinting process indicates a higher material formability [9]. Importantly, in this paper within the range of the considered material properties and temperature the elastic recovery $y_{h}$ is higher than $y_{w}$. It is due to the fact that a higher compression effect occurs between the mold and pattern.

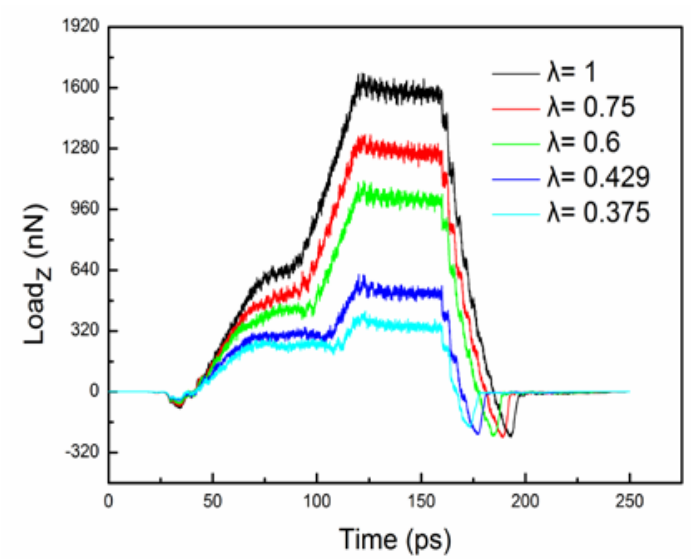

Figure 3. Dependency of the loading force on time for various mold sizes and temperature of $300 \mathrm{~K}$. 


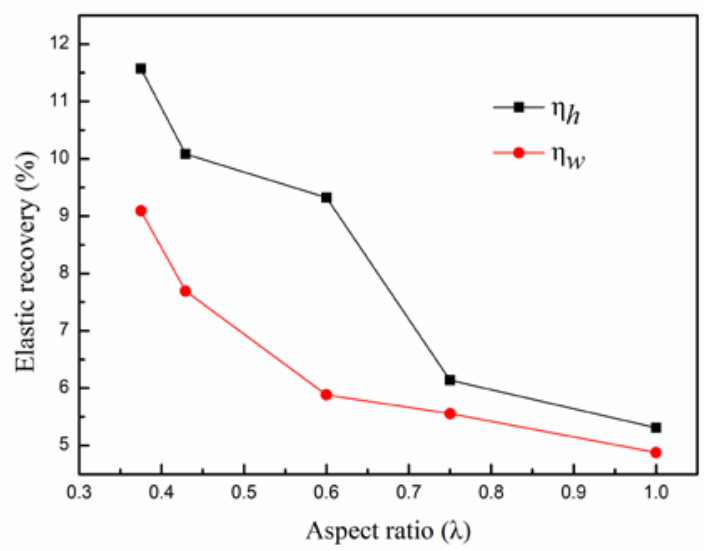

Figure 4. Elastic recovery of NiAl alloys of different mold sizes at temperature of $300 \mathrm{~K}$.

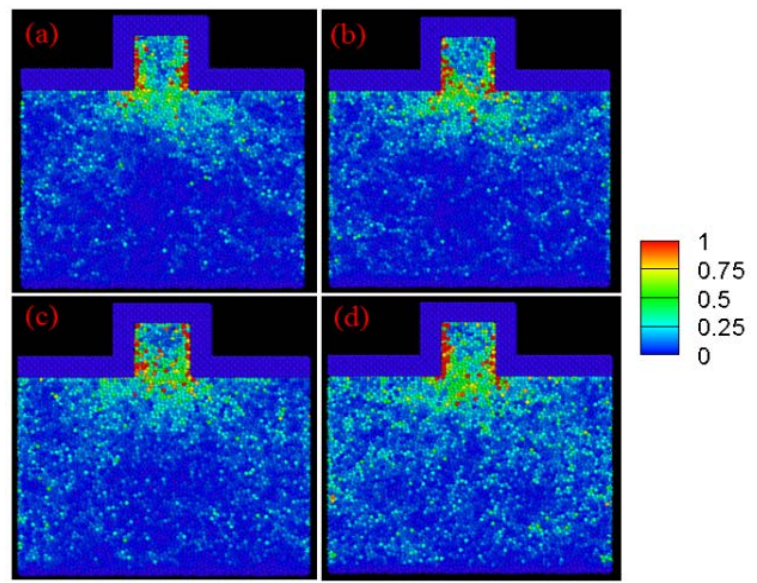

Figure 5. NiAl alloys imprinted at a loading velocity of $30 \mathrm{~m} / \mathrm{s}$ for different temperatures of (a) 300, (b) 500, (c) 700, and (d) $900 \mathrm{~K}$.

Figure 5 presents $\mathrm{NiAl}$ alloys imprinted at a loading velocity of $30 \mathrm{~m} / \mathrm{s}$ for different temperatures of (a) 300, (b) 500, (c) 700, and (d) $900 \mathrm{~K}$, respectively. Deformation mechanism of the amorphous $\mathrm{NiAl}$ alloy is fractal-like with a short-range order at all temperatures. Due to the kinetic energy of pattern atoms, for higher operating temperature the deformation degree of the amorphous alloy is also higher. However, the shear strain distribution does not significantly change within the considered temperature ranges from 700 to $900 \mathrm{~K}$. Moreover, for higher temperatures the degree of softening of the material increases and at high temperatures strain starts gradually extended to the bottom of the material. Figure 6 shows variation of the elastic recovery of amorphous $\mathrm{NiAl}$ alloy for various temperatures at imprinting depth of $3.0 \mathrm{~nm}$ and loading velocity of $30 \mathrm{~m} / \mathrm{s}$. The obtained results given in Fig. 6 indicate that there is a significant difference between the elastic recovery and temperature. This finding is in a good agreement with previous study carried out on the diffusion process of $\mathrm{B} 2 \mathrm{NiAl}$ at high temperature [10], where it was found that diffusion occurred through a variety of cyclic mechanisms, which accomplish the motion of the vacancy through nearest neighbor jumped restoring order to the alloy.

Variation of the elastic recovery of $\mathrm{NiAl}$ alloys imprinted at imprinting velocities of (a) $5 \mathrm{~m} / \mathrm{s}$, (b) $25 \mathrm{~m} / \mathrm{s}$, (c) $30 \mathrm{~m} / \mathrm{s}$ and (d) $50 \mathrm{~m} / \mathrm{s}$ plotted in Fig. 7 indicates that the elastic recovery of the imprinted material decreases by increasing the imprinting velocity. For faster molding process the response time of the material needed to relax stress and deformation reduces. 


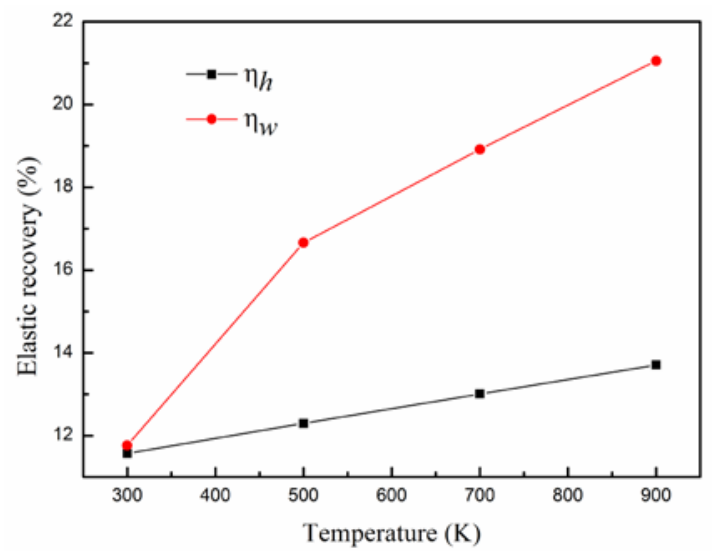

Figure 6. Variation of elastic recovery of amorphous NiAl alloys for different temperatures at the imprinting depth of $3.0 \mathrm{~nm}$ and a loading velocity of $30 \mathrm{~m} / \mathrm{s}$.

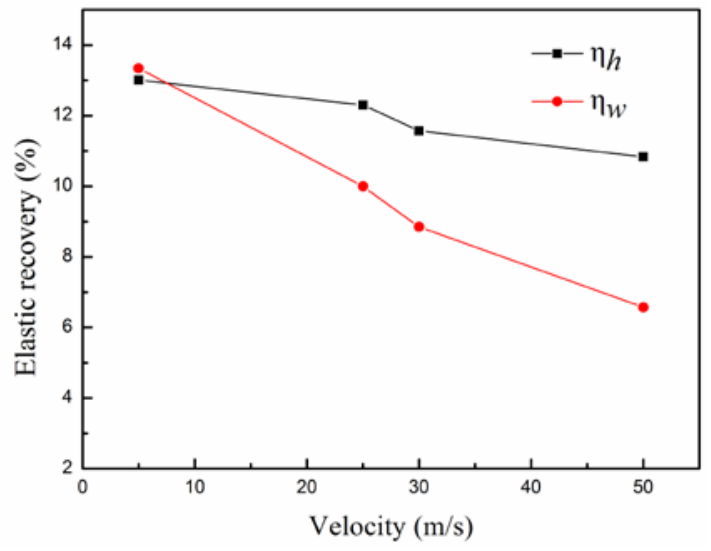

Figure 7. Variation of elastic recovery of NiAl alloys imprinted at imprinting velocities of (a) $5 \mathrm{~m} / \mathrm{s}$, (b) $25 \mathrm{~m} / \mathrm{s}$, (c) $30 \mathrm{~m} / \mathrm{s}$ and (d) $50 \mathrm{~m} / \mathrm{s}$.

\section{Summary}

In this work, MD simulations of amorphous NiAl alloys during imprinting process have been carried out to investigate effects of the materials elastic recovery. A significantly large difference of the shear strain distribution in the inhomogeneous plastic deformation around the mold corner has been found. It has been also observed that the height $\left(\mathrm{y}_{h}\right)$ and width $\left(\mathrm{y}_{w}\right)$ recoveries increase with decreasing the mold aspect ratio. Furthermore, the kinetic energy of pattern atoms causes an increase of the deformation degree of the amorphous alloy with temperature. And, for higher imprinting velocity the elastic recovery of the imprinted material in height and width decreases.

\section{Acknowledgments}

This work was supported by the Ministry of Science and Technology in Taiwan under grants MOST 104-2811-E-151-001, MOST 103-2221-E-151-001 MY3 and MOST 103-2221-E-151-007 MY3.

\section{References}

[1] Q. Zhang, Q.K. Li, M. Li: Acta Materialia Vol. 91 ( 2015), p. 174

[2] Z. Chu, G. Yuan, H. Kato, G. Xie, D. Yan: J. Alloys Compounds Vol. 612 (2014), p. 10

[3] X. Sun, L. Zhuang, W. Zhang, S.Y. Chou: J. Vac. Sci. Technol. B Vol. 16 (1998), p. 3922

[4] T.H. Fang, C.I. Weng: Nanotechnology Vol. 11 (2000), p. 148. 
[5] C.H. Wang, T.H. Fang, P.C. Cheng, C.C. Chiang, K.C. Chao: J. Mol. Model. Vol. 21 (2015), p. 61

[6] F. Cleri, V. Rosato: Phys. Rev. B Vol. 48 (1993), p. 22

[7] C.D. Wu, T.H. Fang, P.H Sung, Q.C. Hsu: Comput. Mater. Sci. Vol. 53 (2012), p. 321

[8] C.D. Wu, T.H. Fang, J.F. Lin: Appl. Surf. Sci. Vol.316 (2014), p. 292

[9] C.D. Wu, T.H. Fang, C.Y. Chen, C.I. Weng: Appl. Surf. Sci. Vol.292 (2014), p. 500

[10] B. Soule De Bas and D. Farkas: Acta Materialia Vol. 51 (2003), p. 1437 